\title{
Endoscopic Anatomy of the Middle Ear
}

\author{
Daniele Marchioni · Gabriele Molteni • \\ Livio Presutti
}

Received: 14 December 2009/Accepted: 31 January 2010/Published online: 23 February 2011

(C) Association of Otolaryngologists of India 2011

\begin{abstract}
Good knowledge of anatomy is fundamental for every surgeon. Middle ear anatomy is really complex and sometimes is challenging for otologists, who need to explore every single compartment for a radical removal of pathology. With introduction of the endoscope in middle ear surgery, anatomy of middle ear spaces has become wider and clearer due to a better magnification and to the possibility to look "behind the corner". This article is a review of the state-of-art of endoscopic middle ear anatomy with description of every compartment, with particular attention to ventilation pathways and middle ear folds.
\end{abstract}

Keywords Middle-ear · Anatomy · Endoscope

\section{Introduction}

In the last 30 years endoscope has been used in endonasal surgery with an increasing number of treatment that nowadays are simple and common [1]. Until the end of nineteenth century development of endoscopic surgery came from urologists, who started endoscopic exploration of the bladder with Max Nitze (1849-1906) [2]. First endoscope was probably described by a German physician born to an Italian family, named Philipp Bozzini, two centuries ago [3]. The development of endoscopic techniques in recent time gave rise to a new application of this tool: endoscopic surgery of middle ear. Since the last decade endoscope was

D. Marchioni · G. Molteni $(\bowtie) \cdot$ L. Presutti

Department of Otolaryngology-Head and Neck Surgery, University of Modena and Reggio Emilia, Azienda Ospedaliero-

Universitaria, Policlinico di Modena, via del Pozzo 71,

41124 Modena, Italy

e-mail: gabriele.molteni@yahoo.it used in otology just as a diagnostic tool and never over the eardrum. Recent development of endoscopy in middle ear surgery [4-11] has permitted an extremely and unexpected detailed view of the "in vivo" anatomy of middle ear. Exploration of hidden recesses like the sinus tympani, the anterior epitympanic space, and the protympanic space with such a magnification is almost impossible with microscopic traditional approaches. Other than a wider and clearer view of middle ear anatomy, endoscopy allows us to better understand middle ear physiology and ventilation pathways that might cause pathology if impaired. In our experience endoscopy allows us not only to perform a less invasive surgical approach, but also a more "physiologic" surgery of middle ear. The aim of this article is to review the state of art of anatomy of middle ear, with particular attention to the epitympanic and retrotympanic spaces. We describe also our experience of how anatomy of middle ear can be improved through an endoscopic view.

\section{Retrotympanic Spaces}

The retrotympanum is a complex structure consisting in different spaces lying in the posterior aspect of the tympanic cavity. Different Authors studied this particularly anatomical region because microscopic view alone is inadequate to explore these posterior spaces.

We can identify four spaces in the retrotympanum: two spaces lying medial and anterior and two spaces lying lateral and posterior to the third portion of the facial nerve and the pyramidal eminence.

The pyramidal eminence is the fulcrum of the retrotympanum, from this structure arise two bony structures: the chordal ridge extending outwards and transversally towards the chordal eminence separating the posterior 
spaces into the facial recess superiorly and the lateral tympanic sinus inferiorly, and the ponticulus ridge extending inwards and transversally to the promontory region dividing the anterior spaces into the sinus tympani inferiorly and the posterior tympanic sinus superiorly.

The sinus tympani (ST) lies medial to the pyramidal eminence, stapedius muscle and facial nerve and it lies lateral to the posterior semicircular canal and vestibule. The superior limit of this space is represented by the ponticulus, instead the inferior anatomical limit is represented by a prominent ridge extending from the styloid eminence to the posterior rim of cochlear window niche: the subiculum (Fig. 1).

The sinus has a great variability in size and shape. Meckel [12] described the sinus tympani for the fist time but he only mentioned the portion anterior to the pyramidal eminence.

Then Steinbrugge [13] studied the depth of the sinus tympani and this Author was the first to describe the posterior extension of the sinus tympani: he probably found a deep sinus tympani with a posterior extension medial to the pyramidal eminence and facial nerve. Observing the variation in depth of the sinus tympani he pointed out the importance of the disease in this kind of sinus tympani. Also Donaldson et al. in the 1970s studied morphology and variations of the sinus tympani. He described a deep sinus tympani laying medial and posterior to the vertical portion of the facial nerve canal; he observed that when the sinus tympani is large it cannot be adequately cleaned with any known instruments by the microscope.

During cholesteatoma surgery a good exposition of the medial boundary of the sinus tympani is very important, because of two important risks. One is the potential persistence of disease inside the sinus due to incomplete removal and the second is the increased risk for ossicular discontinuity and hearing loss due to cholesteatoma within the ST [14, 15], which the surgeon cannot control [16].

To avoid these risks, maximum exposure of the ST and complete removal of the disease are essential. Traditional surgical techniques, like canal wall down and canal wall up tympanoplasty, have been demonstrated to fail to provide direct access to the ST and blind instrumentation is required to remove the disease [17]. A posterior access to the ST through the mastoid has been suggested as a possible alternative solution [18] even if this approach presents several disadvantages and requires an expert otosurgeon. Recent introduction of the endoscope during the surgical procedure allow us to have a good exposition of the sinus tympani and posterior sinus.

Thomassin et al. [5] observed that the surgical control of the entire sinus tympani is possible using the endoscope and he found that the quality of disease eradication had significantly improved with intra-operative use of the endoscope, with a consequent reduction of residual cholesteatoma. The authors suggested the use of endoscopes with different angles to explore the sinus tympani; especially 30,45 and $70^{\circ}$ telescopes allow a better control of the middle ear cavity. Badr-El-Dine [6] and El-Meselaty et al. [19] studied utility of the endoscope during cholesteatoma surgery and they found that endoscopic approach to sinus tympani gave a better exposition of the sinus thus achieving better pathology eradication.

Baki et al. [4] studied the endoscopic anatomy of the sinus tympani on 30 cadaveric temporal bones. They found that the sinus tympani is bounded laterally by a constant ledge of bone anterior to the facial nerve. They found a deep extension of the sinus tympani posterior to the facial nerve in six specimens: in these cases it wasn't possible to clearly visualize this region also with endoscope because the orifice plane was perpendicular to the external auditory canal axis. They suggested removal of a lateral rim of bone anterior to the facial nerve to allow a good exposure of the deep sinus tympani with an optical instrument.

Recently we studied the endoscopic anatomy of the sinus tympani and the feasibility of the endoscopic approach to the sinus tympani. In our study we performed 40 endoscopic approaches to the sinus tympani in subjects affected by a sinus cholestetoma. We classified the morphology shape of sinus tympani on the basis of the intraoperative finding and we observed anatomical variations of the ponticulus. This is possible using a particular position of the surgeon during the endoscopic approach. In fact, position of the surgeon during endoscopic approach to the sinus tympani is different from traditional microscopic surgery: the surgeon stand on the opposite side of the affected middle ear to permit a better view of the anatomy
Fig. 1 a-b Endoscopic anatomy of the sinus tympani during a transcanal approach. st Sinus tympani, $p$ ponticulus, $s u$ subiculum, $r w$ round window, $p r$ promontory, ps posterior sinus, $m a$ malleus, $s$ stapes, in incus, pe pyramidal eminence, $f n$ facial nerve
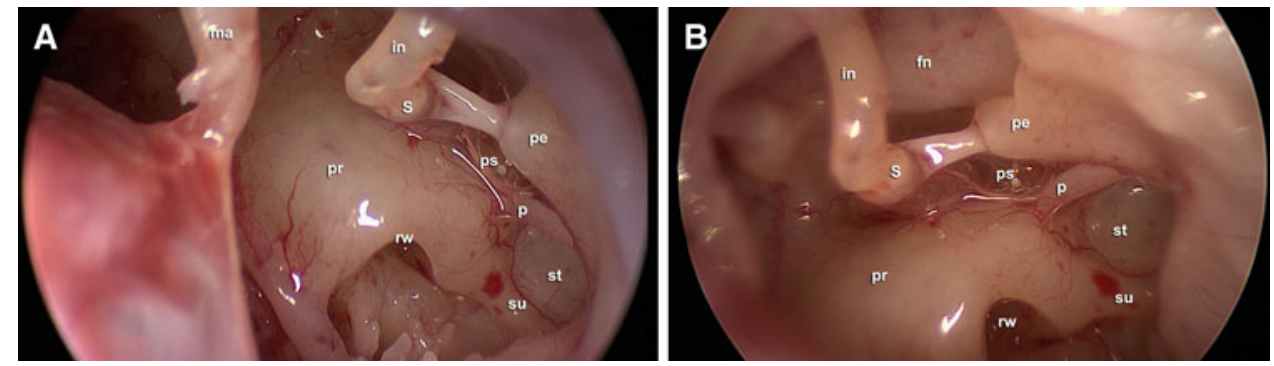
of the sinus tympani area. Introducing the endoscope into the external ear canal with $45^{\circ}$ angle from the opposite site, a wide view of the medial boundary of the sinus tympani and of the ponticulus area is permitted. With this technique when pathology is present in the sinus tympani we are able to remove it using a suitable angled instrument.

Based $o$ the shape of the ST and thank to a wider endoscopic view of this region, we classified ST in different types (Fig. 2).

- Classical shape: when the sinus is located between the ponticulus and subiculum lying medial to the facial nerve and to the pyramidal process (Fig. 2a).

- Confluent shape: when an incomplete ponticulus is present and the ST is confluent to the posterior sinus (Fig. 2b).

- Partitioned shape: when a ridge of bone extending from the third portion of the facial nerve to the promontory area is present, separating the sinus tympani into two portions (superior and inferior) (Fig. 2c).

- Restricted shape: when a high jugular bulb is present thus reducing the inferior extension of the sinus tympani (Fig. 2d).

We described for the first time the sinus tympani with a partitioned shape and we found that the bony crest arise from the third portion of the facial nerve could be present like a ridge or like a bridge of bone, presenting or not a communication between the superior and the inferior portions of the sinus. This is very important because in one subject we found a cholesteatoma under a partitioned shape sinus tympani and we had to remove the bony crest endoscopically in order to completely remove the disease.

The endoscopic approach to the ST permits also a good view of the ponticulus. It is a bony ridge extending from
Fig. 2 Different morphologic types of the ST. a classical shape, $\mathbf{b}$ confluent shape, c partitioned shape, $\mathbf{d}$ restricted shape. st Sinus tympani, $p$ ponticulus, $r w$ round window, $p r$ promontory, $p s$ posterior sinus, $s$ stapes, $s u$ subiculum, $p e$ pyramidal eminence, $f n$ facial nerve, $j b$ jugular bulb, *ridge of bone separating the sinus tympani
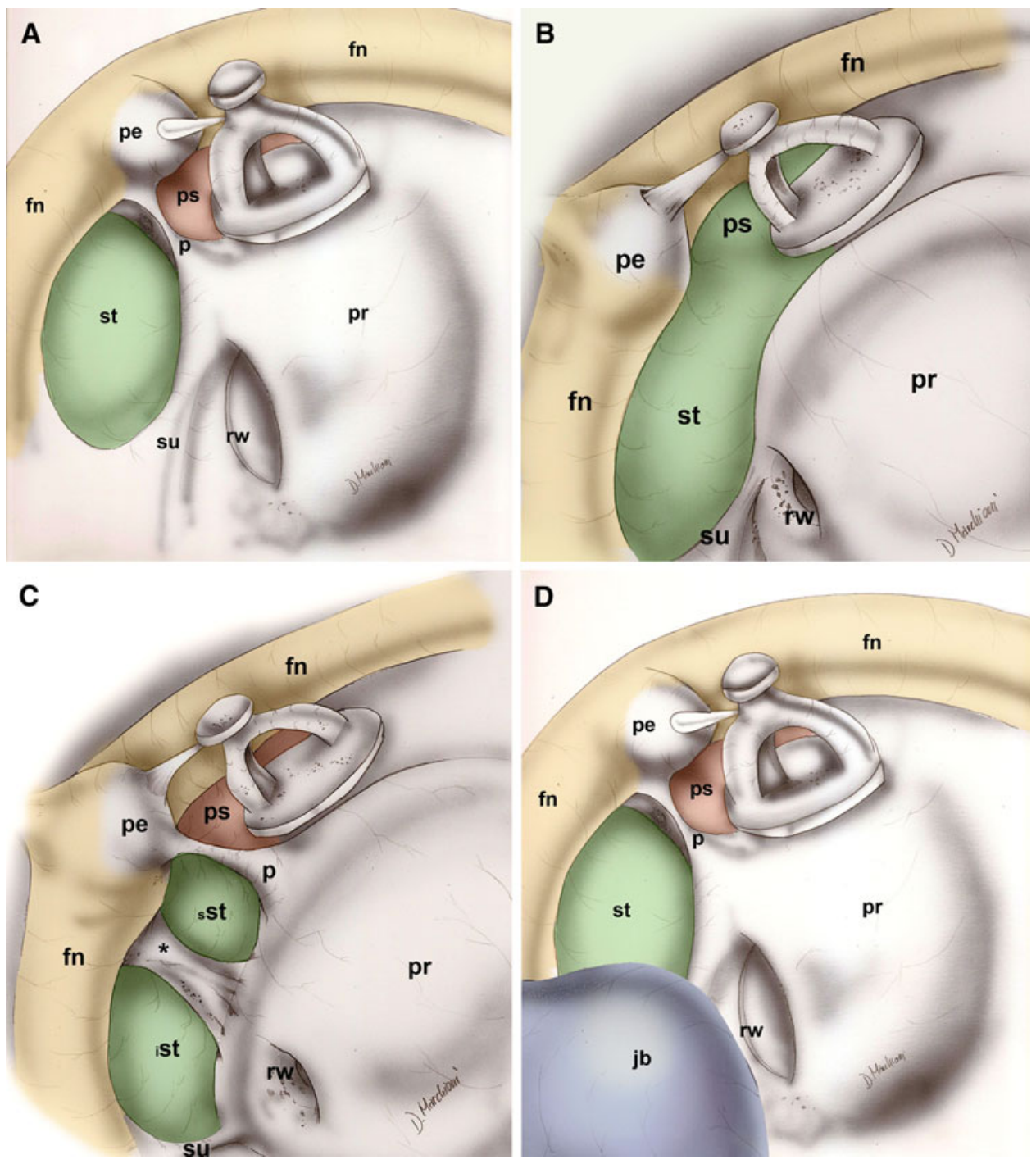
the pyramidal process to the promontory region, which separates the sinus tympani from the posterior sinus, representing the superior limit of the ST. Few studies in literature focus on this anatomical structure. Holt [20] observed the anatomy of the ponticulus in a dissection study on 50 cadaveric temporal bones. In this study the Author found a complete ponticulus in 33 cases, a partial ponticulus in seven, and a complete absence of it in ten bones.

In our study, the endoscopic approach to the sinus tympani with a $45^{\circ}$ instrument allowed us to visualize the morphology of the ponticulus area in the majority of subjects (38 of 40 cases).

The endoscopic approach allowed us to find three different variants of the ponticulus (Fig. 3).

- Classical morphology: in these patients the ponticulus is completely formed and it is like a ridge of bone extending from the pyramidal process to the promontory area; this structure represent the superior limit of ST dividing it from posterior sinus (Fig. 3a).

- Incomplete ponticulus: in these cases, ST and posterior sinus are confluent (Fig. 3b).

- Communicating ponticulus: in these subjects, the ponticulus is like a small bridge of bone and there's a communication between ST and posterior sinus under it (Fig. 3c).

Especially when ponticulus is like a small bridge intraoperative endoscopic evaluation of the ponticulus area is very useful, because a residual cholesteatoma could be present under the bony bridge. In our series we found residual cholesteatoma under the ponticulus in two subjects and in one patient we had to remove the ponticulus by drilling the bone, in order to remove the residual cholesteatoma under endoscopic control.

Several anatomical studies focused on the depth of ST. This detail is very important because the more the ST is deep the more a radical removal of cholesteatoma is difficult to achieve. Persistence of pathology is among the major cause of failure in surgery of cholesteatoma. We studied the frequency of residual cholesteatoma detected with the intra-operative use of endoscope after traditional microscopic surgery and we found that the sinus tympani is the most common site of residual cholesteatoma fragments [21]. This is confirmed by other studies reported in international literature, which showed the same results. This is particularly true especially when the sinus tympani is deep [19-22]. For this reason it might be very useful for the surgeon to study the extension of the ST before the surgery. In our recent study we propose a morphological classification of the ST using computed tomography (CT) [23]. We assessed the variation in depth of the sinus tympani area, analyzing the posterior and medial extension of the medial boundary of the ST based on analysis of axial slices of ST. We believe that this classification could be useful for the surgeon in the pre-operative planning of the type of approach to ST.

Based on radiological findings we classified the depth of the ST into three types as follow (Fig. 4).

Type A: small sinus tympani. The medial limit of the third portion of the facial nerve corresponded to the depth of the sinus). In these cases, the sinus tympani is small and don't present a medial and posterior extension to the facial nerve (Fig. 4a).

Type B: deep sinus tympani. The medial boundary of the ST lies medially with respect to the third portion of the facial nerve and don't present a posterior extension to the facial nerve (Fig. $4 \mathrm{~b}$ ).

Type $C$ : deep sinus tympani with posterior extension. The medial boundary of the ST lies medial and posterior to the third portion of the facial nerve. In these cases, ST is very large and deep and all these patients have a well-developed mastoid. (Fig. 4c).

In our experience, the transcanal endoscopic approach is indicated in patients with sinus tympani type A-B. In our preliminary study it was possible to explore the depth of the ST and remove the pathologic tissue from the cavity in a safety way in 33 out of 35 patients.

When the patient has a type C ST it is not always possible to explore the entire depth of the sinus, especially when it is associated with a well-developed mastoid cells. In these cases it was necessary to perform a posterior retrofacial approach.
Fig. 3 Different morphologies of ponticulus. a classical morphology, b incomplete ponticulus, c communicating ponticulus. st Sinus tympani, $p$ ponticulus, $r w$ round window, $p r$ promontory, $p s$ posterior sinus, $s$ stapes, pe pyramidal eminence, $f n$ facial nerve
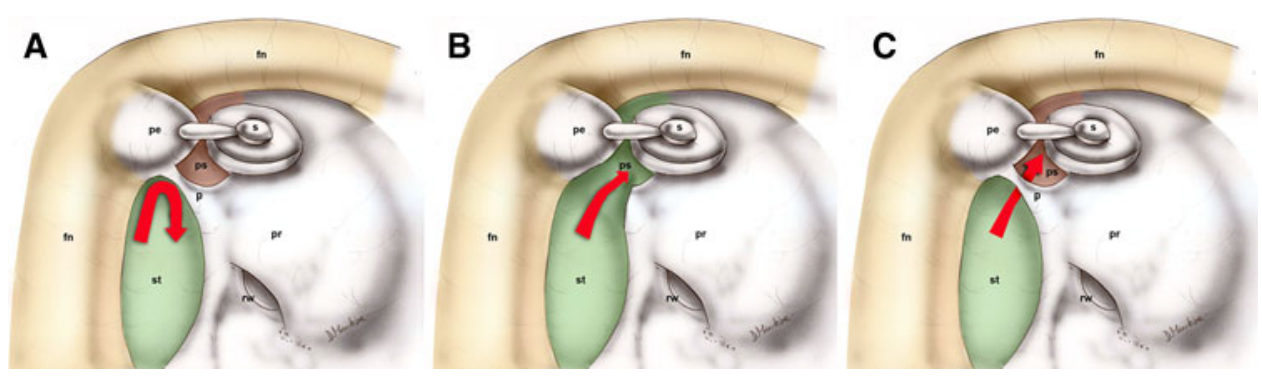
Fig. 4 Classification ST's depth based on axial CT scan. a limited sinus tympani, $\mathbf{b}$ deep sinus tympani with medially extension respect the facial nerve; c deep sinus tympani with posterior extension respect the facial nerve. $f n$ Facial nerve, $p r$ promontory, $r w$ round window, st sinus tympani
Type A
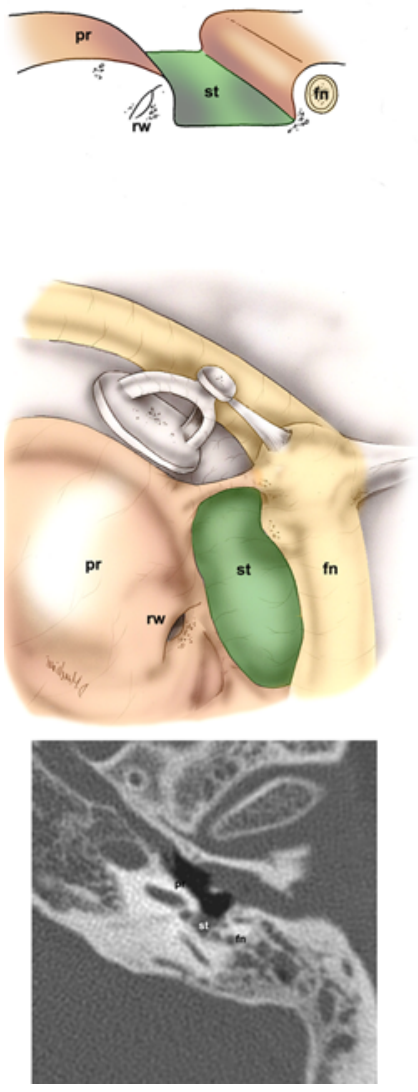

Type B
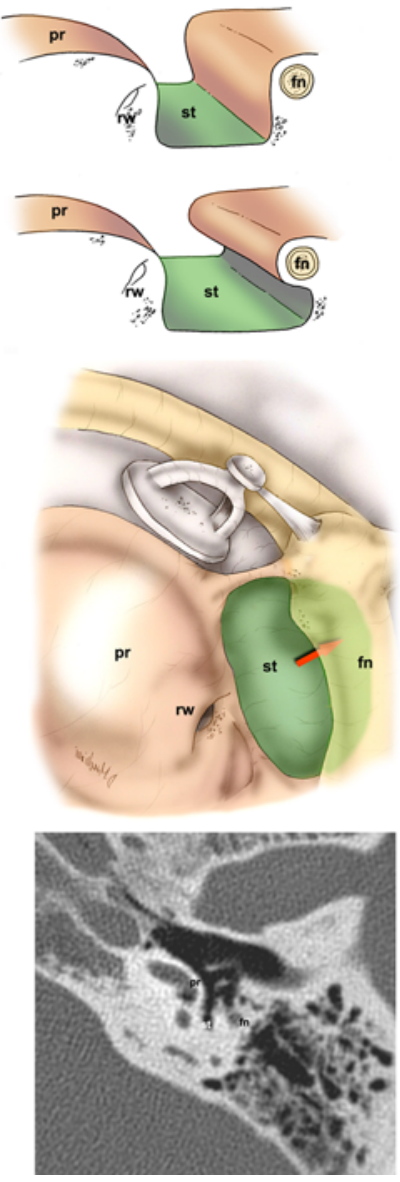

Type C
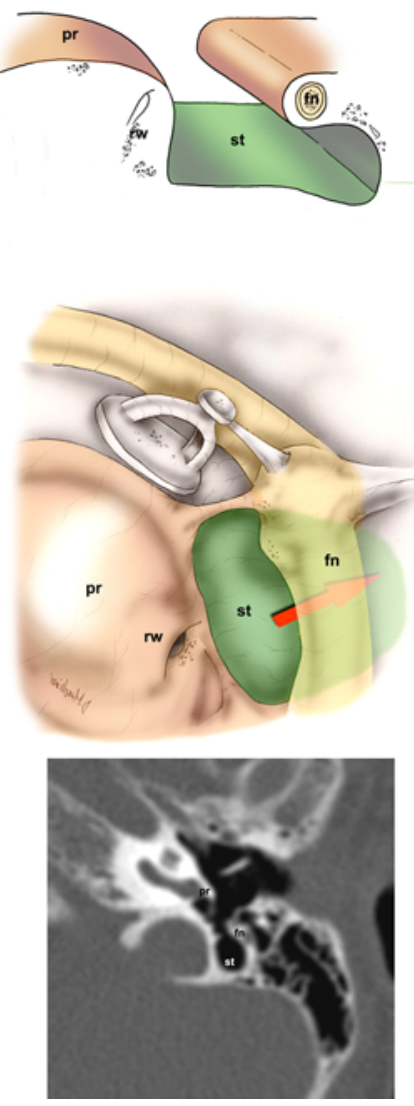

In our experience performing endoscopic middle-ear surgery, close and variable relationships have been noted between ST, posterior tympanic sinus (PTS) and the pyramidal eminence (PE). Pneumatization of the retrotympanum may extend to a variable degree into a recess under the PE. We described this space in an unpublished work and we called this anatomical finding the "subpyramidal space' (SS) (Fig. 5) [24]. This space is limited laterally by the medial aspect of the pyramidal process, medially by the lateral wall of the tympanum, inferiorly by the ponticulus, and posteriorly and superiorly by the Fallopian canal, and it could be in direct anatomical continuity with the ST or with the PTS, depending on the position of the ponticulus. Features of this space (particularly its depth) vary significantly and we found that it could range from a total absence due to a complete development of the medial aspect of the pyramidal process, up to a clear representation of the SS with a significant depth. The more SS is deep, the more a surgical approach is at high risk of leaving residual cholesteatoma. A good knowledge of these anatomical spaces may help in reducing the risk of residual cholesteatoma during middle ear surgery.

\section{Epitympanic Spaces}

The epitympanic space is a pneumatized portion of the temporal bone superior to the mesotympanum. Different Authors studied the anatomy of the epitympanic compartments: recently Palva and coll. described the anatomy of epitympanic diaphragm studying ventilation pathways of the epitympanum [25]. "Epitympanic diaphragm" consists of three malleal ligamental folds (anterior, lateral, and posterior), the posterior incudal ligamental fold and two purely membranous folds (the tensor fold and the lateral incudomalleal fold) together with the malleus and incus. From this anatomical point of view it is possible to classify the epitympanum in two different compartments: a larger and posterior one (posterior epitympanic space-PES) and a smaller and anterior compartment (anterior epitympanic space-AES) (Fig. 6b-c). Body and short process of the incus together with head of the malleus occupy the majority of posterior epitympanic space. The lateral portion of posterior epitympanum is narrower and it is divided in two further portions by the lateral incudo-malleal fold. They are separated and arranged one above the other: the 
Fig. 5 The subpyramidal space. a-b retrotympanic space with endoscope $45^{\circ}$ angle view in a subject with a confluent shape of the ST; c magnification of posterior sinus and subpyramidal space (arrow); d exploration of subpyramidal space laying under the pyramidal eminence. st Sinus tympani, $p r$ promontory, $p s$ posterior sinus, $s$ stapes, pe pyramidal eminence, $f n$ facial nerve, $l c$ lateral semicircular canal, an antrum, ss subpyramidal space, $P E S$ posterior epitympanic space, $c p$ cochleariform process, ma malleus
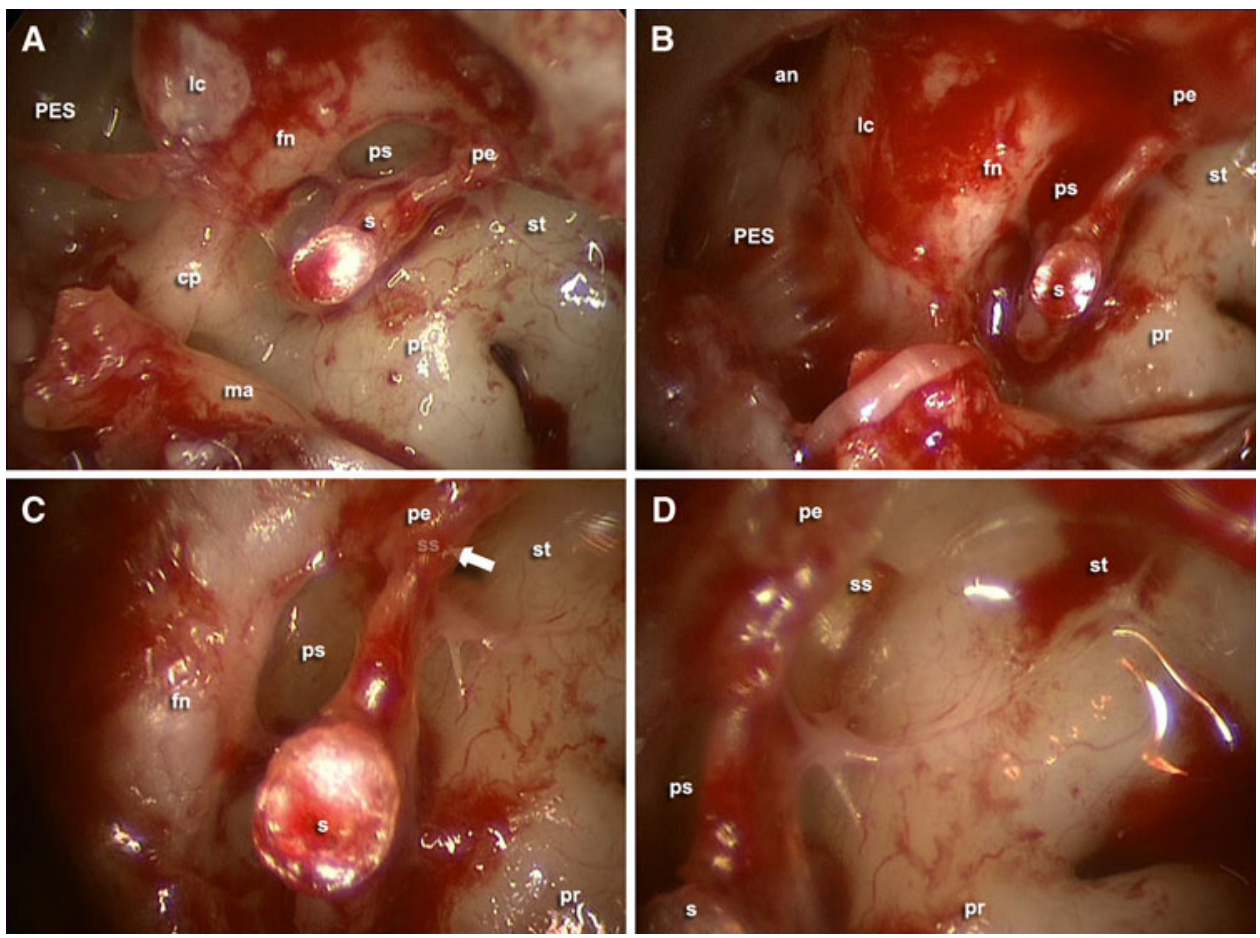

superior and inferior lateral attic (Fig. 6a-c). Inferior lateral attic is bounded superiorly by the lateral incudo-malleal fold and it is located between the more declive portion of short process and body of the incus medially and the medial aspect of the scutum laterally. This anatomical area is therefore in a lower position to the epitympanic diaphragm and is in communication with the underlying mesotympanum. Mesotympanic region guarantees ventilation of the inferior lateral attic. In a more cranial position lies the superior lateral attic whose inferior limit is represented by the incudo-malleolar fold. This anatomical area together with the medial attic is the so-called superior attic or upper unit. Superior attic is in communication with the mesotympanum through the underlying tympanic isthmus and posteriorly it is opened to the aditus ad antrum. Its upper limit is tegmen tympani, while the lower one is given by the second portion (intratympanic) of the facial nerve and laterally it is bounded by the lateral bony wall of the Atticus. The whole superior attic is ventilated through the isthmus (Fig. 7).

Epitympanic compartments receive their aeration via the large tympanic isthmus between the medial part of the posterior incudal ligament and the tensor tendon. Palva et al. observed that the aeration pathway from the Eustachian tube leads directly to the mesotympanic and hypotympanic spaces, whereas the epitympanum is away from the direct air stream and is only aerated through the tympanic isthmus (Fig. 8a-b) [26].

The lower-unit is a reduced compartment represented by the Prussak's space. It is separated anatomically and physiologically from the upper-unit by its vault, which is represented by the lateral malleal ligament fold (Fig. 6a-c). This inferior epitympanic portion is ventilated in the majority of cases from the posterior pocket through mesotympanum (Fig. 8b-c). For this reason the two ventilatory trajectories of the epitympanic units are separated from each other. We strongly believe that this physiological concept is of extreme importance in transcanalar endoscopic middle-ear surgery. This is because restoration of ventilation pathways with unification of the upper and the lower-unit through the creation of a large tympanic isthmus and an accessory route through the tensor fold is the basis of this surgery. Surgery must ensure ventilation to all parts of epitympanum.

Posterior epitympanic space contains the incudo-malleolar joint. This articulation is a crucial landmark also during the transcanal endoscopic approach. When the endoscopic surgeon has to deal with an epitympanic involvement of cholesteatoma, he needs to remove the scutum by drilling the lateral bone in the attic, discovering the body of the incus and the head of the malleus. Short process of the incus shows us the antrum's location (Fig. 9a-b) and drilling the bone over the short process we can go directly to the antrum without any problem (Fig. 9c-d). In this way we are able to endoscopically remove epitympanic pathologic tissue with antrum involvement. In some the ossicular chain is not present into the epitympanic compartment and thus we need other anatomical landmarks. In our experience we use an anatomical triangle as a landmark in these complex cases 
Fig. 6 Epitympanic spaces and their ventilation routes.

a posterior view; $\mathbf{b}$ medial to laterally view; c: lateral view. Long curved arrow ventilation route of the epitympanicmastoid compartments, short arrow ventilation route of the Prussack space, ma Malleus, in incus, $s$ stapes, $c p$ cochleariform process, AES anterior epitympanic space, $P E S$ posterior epitympanic space, pos posterior spine, $e t$ Eustachian tube, imlf lateral incudomalleal fold, $m l f$ lateral malleal fold, $t f$ tensor fold, $\mathrm{plm}$ posterior malleal ligamental folds, alm anterior malleal ligamental folds

\section{A}

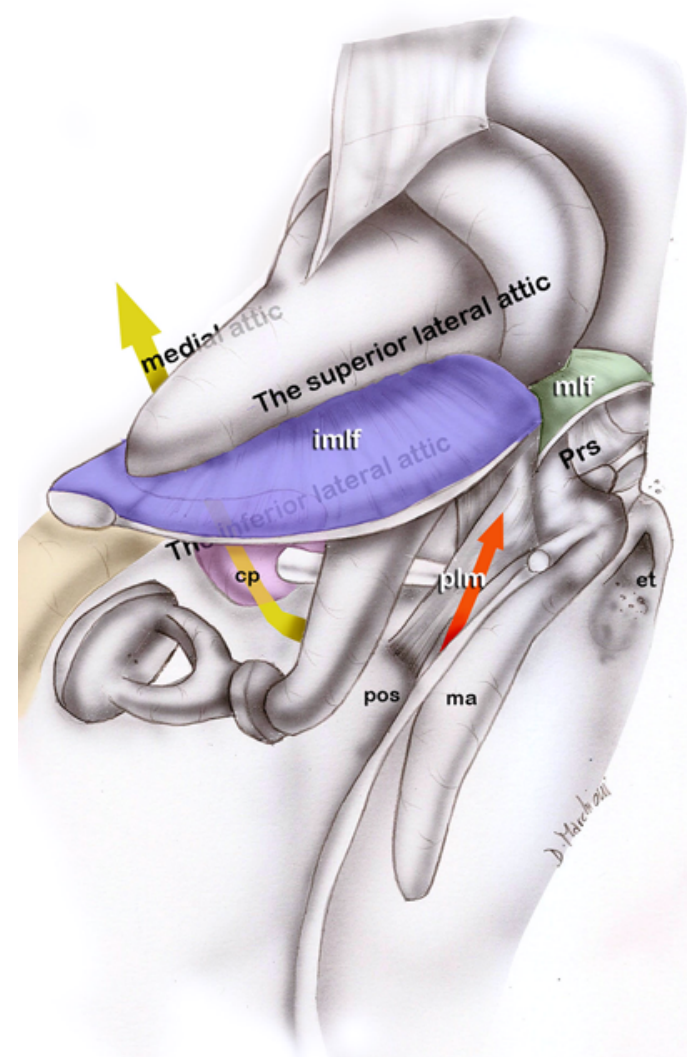

B

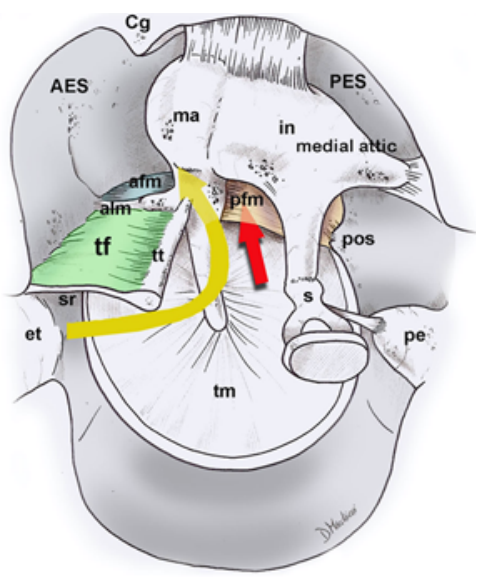

C

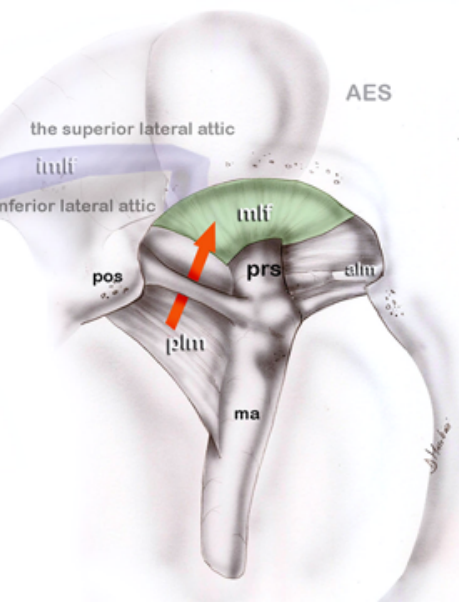

Fig. 7 Tympanic isthmus. a magnification of the isthmus with a $0^{\circ}$ endoscope;

b magnification of the isthmus with a $45^{\circ}$ angle endoscope; c scheme of the isthmus. ma Malleus, in incus, $s$ stapes, $c p$ cochleariform process, $t t$ tensor tendon of the malleus, $P E S$ posterior epitympanic space, $c t$ corda tympani, tf tensor fold, $f n$ facial nerve, pe pyramidal eminence, $l c$ lateral semicircular canal

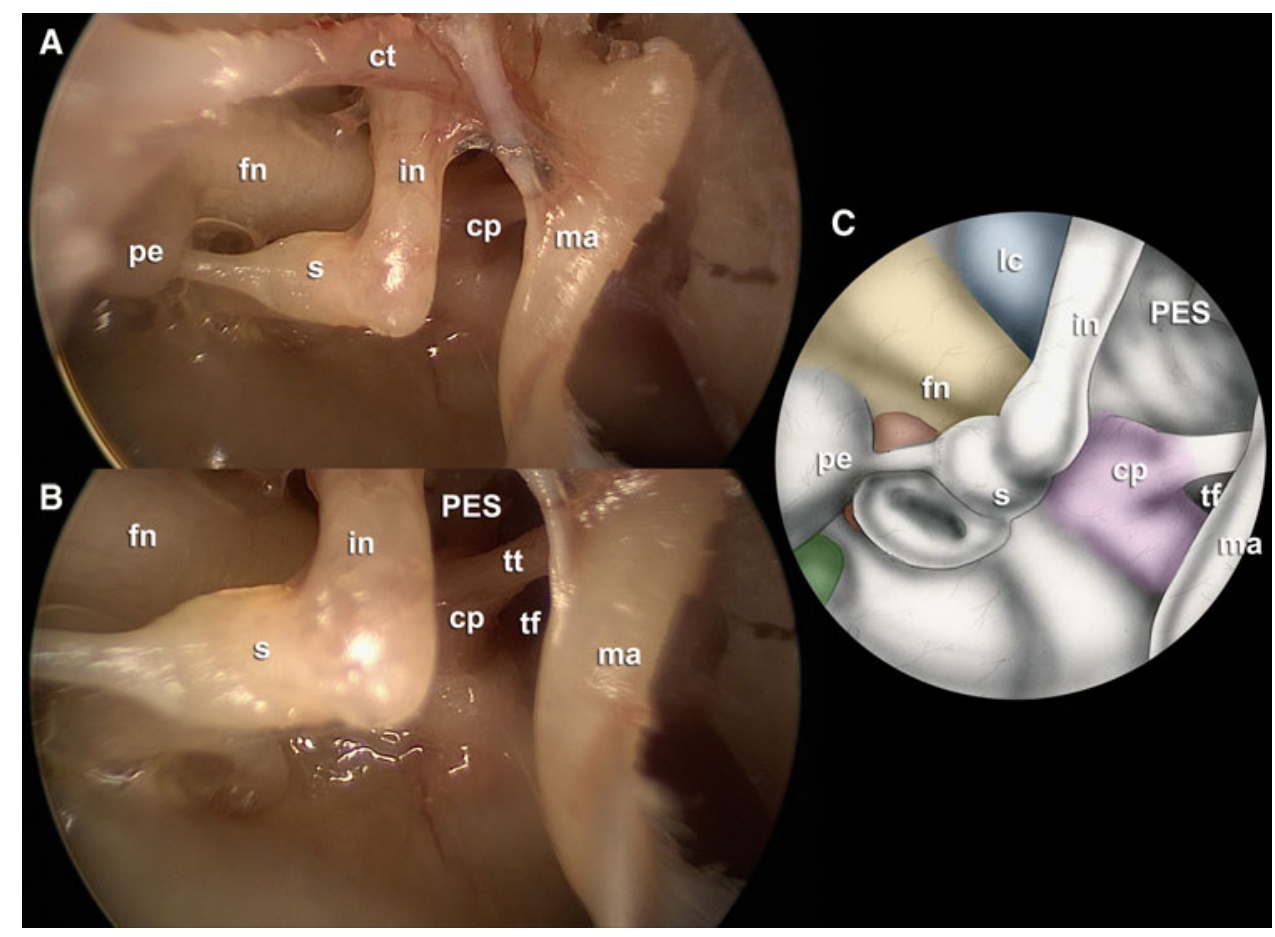


Fig. 8 The two independent ventilation routes of the middle ear. $\mathbf{a}-\mathbf{b}$ ventilation route to the epitympanic compartments through the isthmus (long arrow); c-d ventilation route to the Prussak's space through the posterior pouch (short arrow). ma Malleus, hma head of the malleus, in incus, $s$ stapes, $c p$ cochleariform process, $A E S$ anterior epitympanic space, $P E S$ posterior epitympanic space, pos posterior spine, $e t$

Eustachian tube, imlf lateral incudomalleal fold, $\mathrm{mlf}$ lateral malleal fold, $t f$ tensor fold, $p l m$ posterior malleal ligamental folds, alm anterior malleal ligamental folds, $p r$ promontory, $r w$ round window, scu scutum, prs prussack space, tm tympanic membrane, af anterior fold of the malleus
A

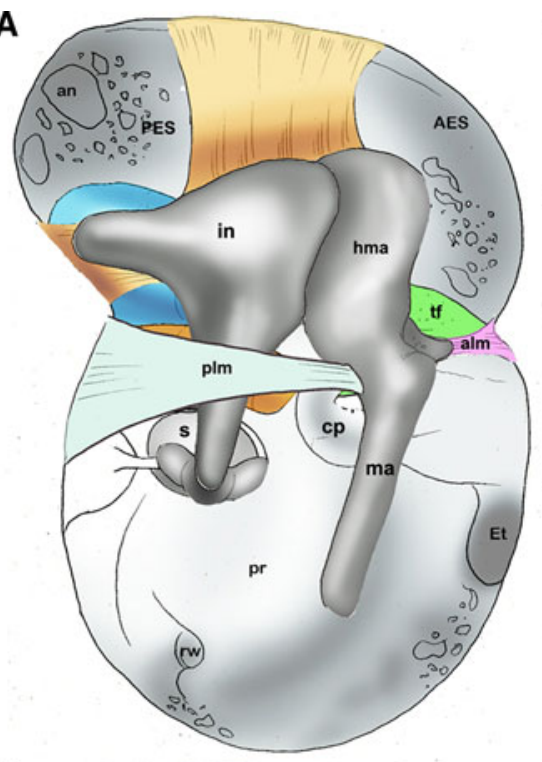

C

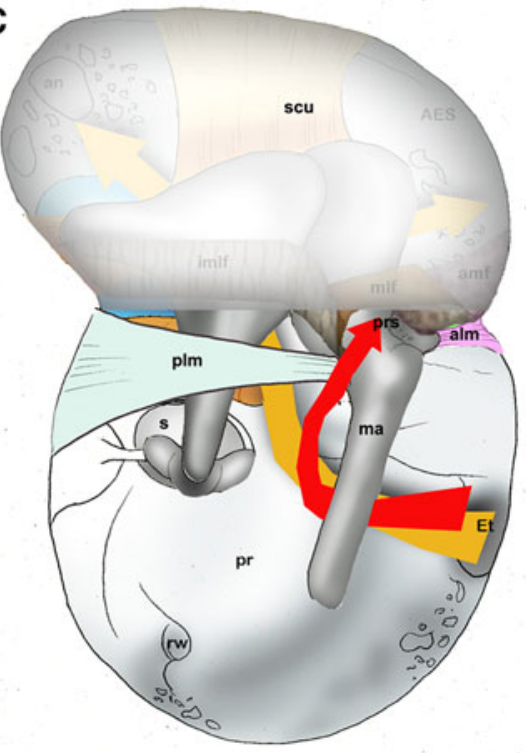

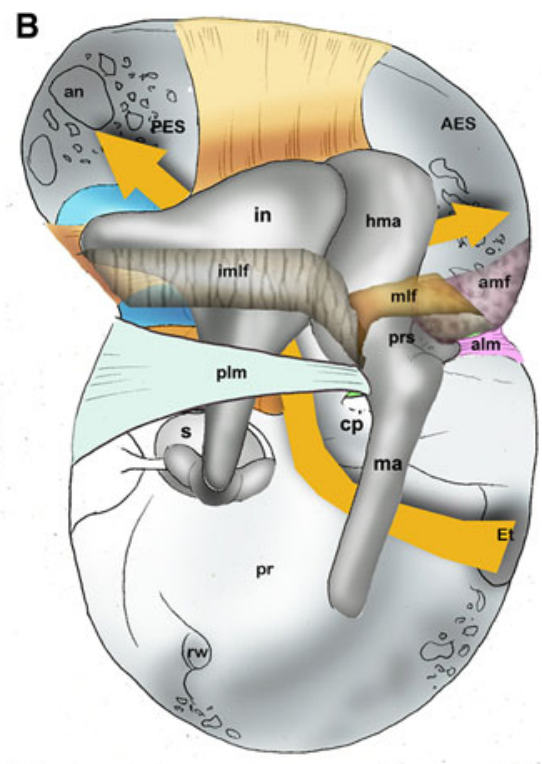

B

D

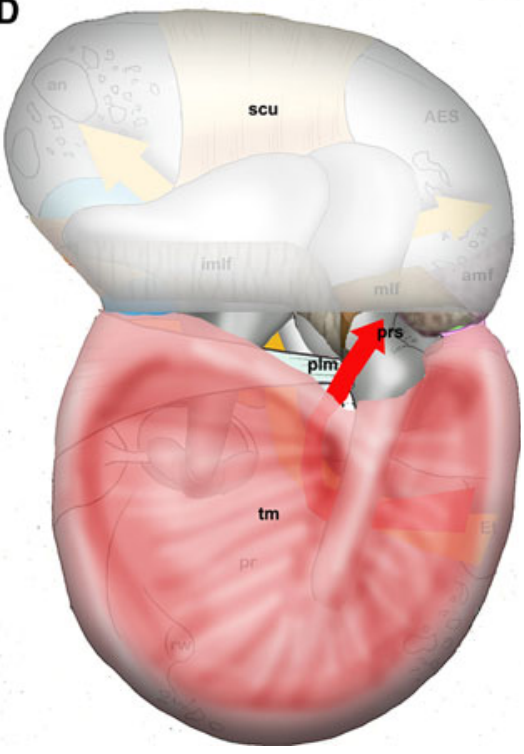

(Fig. 10). The superior side of this triangle is tympanic tegmen, bony wall of the AES draws the anterior side and the inferior side is the lateral semicircular canal wall. Tip of the triangle show us where is the antrum and the surgeon can drill in a safety way until the antrum is visible (Fig. 10c-d). During transcanal approach we need to look for the second tract of the facial nerve in order to find the lateral semicircular canal: it usually lies superior and posterior to the facial nerve (Fig. 10a-b).

The AES has attracted the attention of ear surgeons because of its relationship to important surrounding structures and to its frequent involvement by cholesteatoma. Demarcation between the anterior and posterior epitympanum is represented by the transverse ridge or " $\operatorname{cog}$ ". The $\operatorname{cog}$ is a bony septum that detaches from the tegmen tympani cranially, heading vertically toward the cochleariform process, in front of the malleus head. W. House was the first to describe the $\operatorname{cog}$, this bony crest lying anterior to the head of the malleus, representing an embryologic remnant of the fusion plane between saccus anticus and medius, separating the anterior epitympanic space from the posterior epitympanic space.

Different surgeons reputed this anatomical structure an important landmark for identifying the second tract of the facial nerve during microscopic transmastoid approach. Instead, during transcanal endoscopic approach the second tract of the facial nerve is ahead toward the surgeon's instrument. The endoscope allows us to see directly the structures representing the floor of the epitympanum: the second tract of facial nerve, the cochleariform process and the tensor fold.

Especially the cochleariform process and the tensor fold with the tensor tympani canal represent the floor of the 
Fig. 9 Endoscopic dissection view. Short process of the incus is a landmark for the antrum. a left ear after atticotomy with uncovered incudo-malleolar joint; $\mathbf{b}$ drilling the posterior portion of the scutum over the short process of the incus; c short process of the incus showing where the antrum is located; $\mathbf{d}$ relationship between the antrum and the short process of the incus after removing attic's lateral bony wall. $m a$ Malleus, hma head of the malleus, in incus, $f n$ facial nerve, $c t$ corda tympani, an antrum, $s p l$ short process of the incus
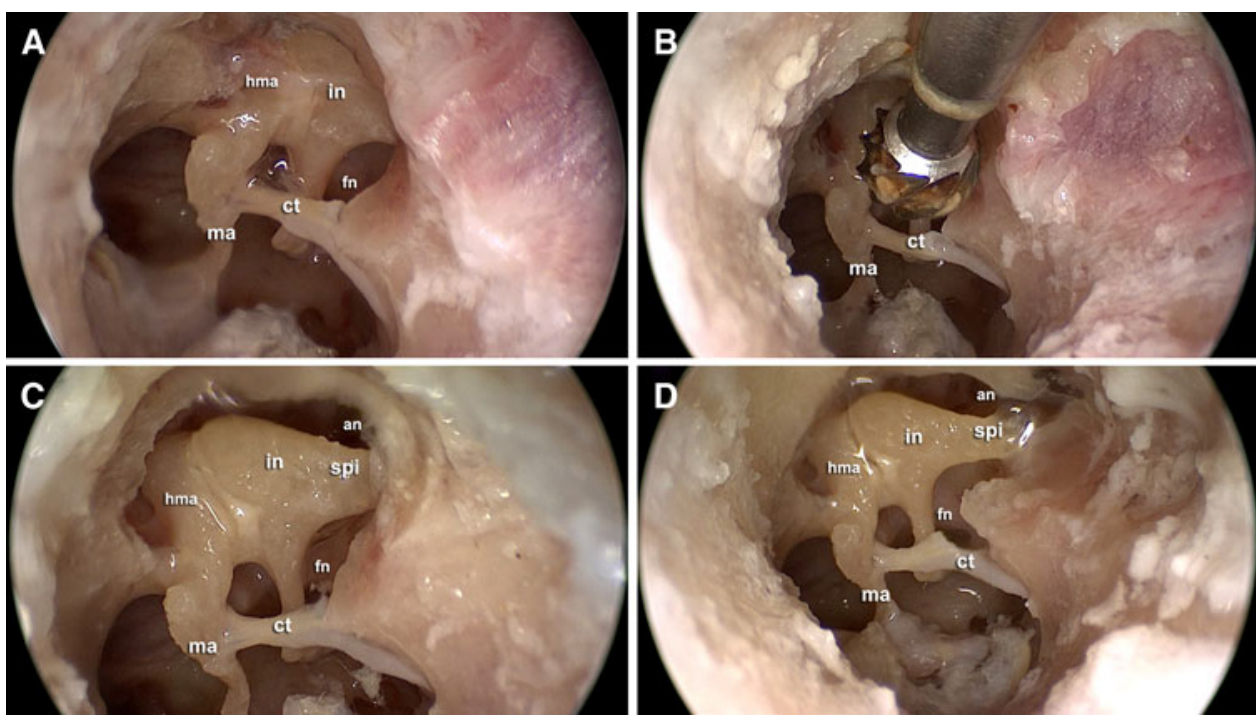
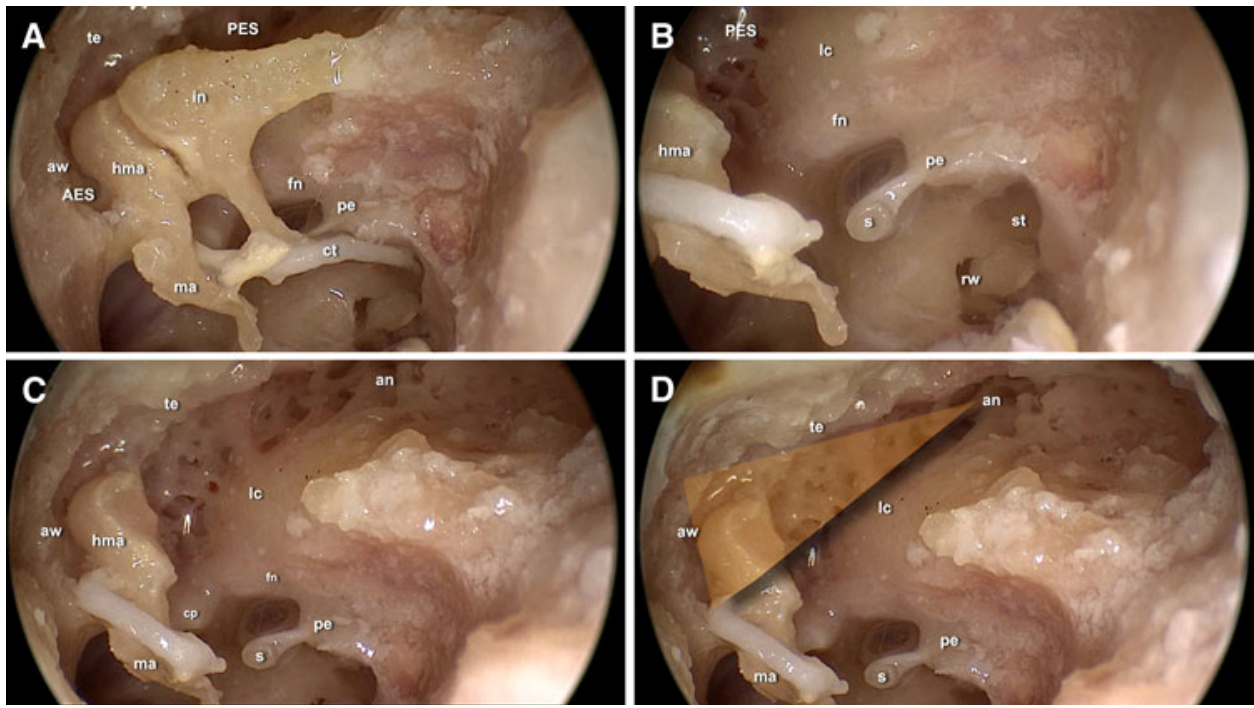

Fig. 10 Endoscopic dissection demonstrating the epitympanic triangle. a left ear after removing attic's lateral bony wall; b incus removal. Second tract of the facial nerve is a landmark for the lateral semicircular canal (it lies superiorly and posteriorly to the second tract of the facial nerve); $\mathbf{c}-\mathbf{d}$ limits of the triangle defined superiorly by the tegmen tympani, anteriorly by the anterior bony wall of the

anterior epitympanic space (Fig. 11). AES is limited anteriorly by the root of zygomatic arch (a thick bony plate which separates it from pericarotic cells), superiorly by the tegmen tympani (that separates it from the dura mater), laterally by the tympanic bone and chorda tympani, and medially by a bony wall that separates it from the genicolate fossa, which contains the homonymous ganglion. The tensor fold that separates it from the underlying sovratubaric recess when completed represents its inferior limit.

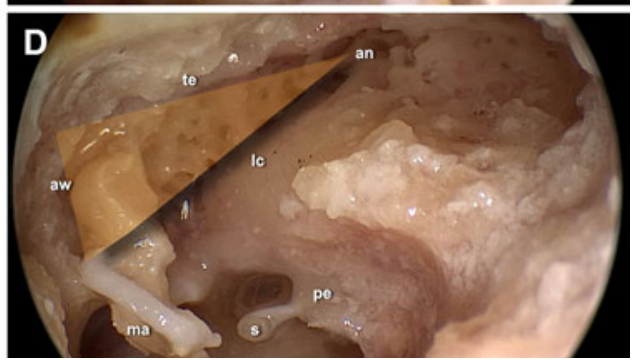

AES and inferiorly by the lateral semicircular canal orientation. Tip of the triangle shows were the antrum is located. ma Malleus, hma head of the malleus, in incus, $s$ stapes, $f n$ facial nerve, ct corda tympani, an antrum, pe pyramidal eminence, $P E S$ posterior epitympanic space, $A E S$ anterior epitympanic space, $l c$ lateral semicircular canal, te tegmen, aw anterior bony wall of AES

Few articles studied the variation in shape and size of the AES; Onal et al. studied 30 human temporal bone using two different methods: 20 bones were cut vertically and in ten bones was performed a modified radical mastoidectomy [21]. They found that the AES embryologically sowed two kind of morphology (Fig. 12c). AES type I (83\%) consists of two cavities separated by the tensor tympani fold developing from the anterior saccule of the saccus medius and saccus anticus, which might be called the anterior mallear space, the cavity superior to the tensor fold, 

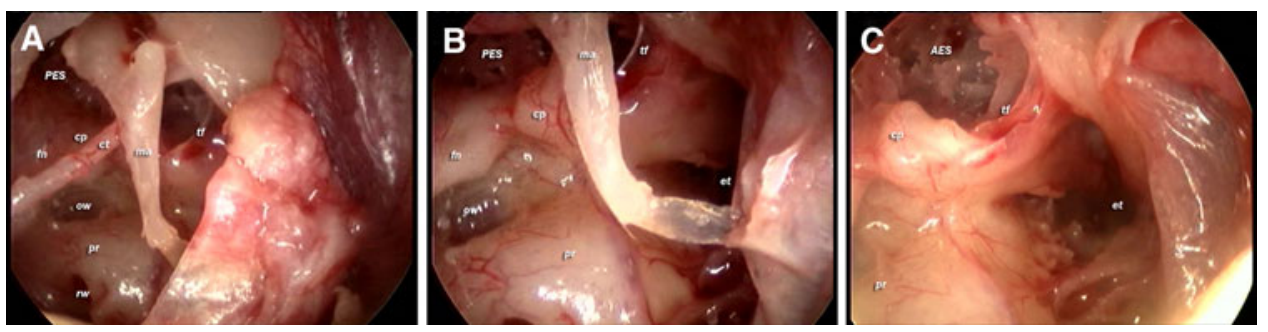

Fig. 11 Endoscopic dissection showing the floor of epitympanic compartments (second tract of facial nerve, cochleariform process and tensor fold) after incus and stapes removal. a $45^{\circ}$ endoscope view showing an incomplete tensor fold with a direct communication from the protympanic space to the anterior epitympanic space; $\mathbf{b}$ magnification of the tensor fold area with a $45^{\circ}$ endoscope; $\mathbf{c}$ anatomy of the tensor fold after malleus removal. This picture shows the relationship between the protympanic space and the AES through the tensor fold. $m a$ Malleus, $s$ stapes, $f n$ facial nerve, $c t$ corda tympani, $P E S$ posterior epitympanic space, $A E S$ anterior epitympanic space, if tensor fold, $p r$ promontory, et Eustachian tube, $c p$ cochlear process, $r w$ round window, ow oval window

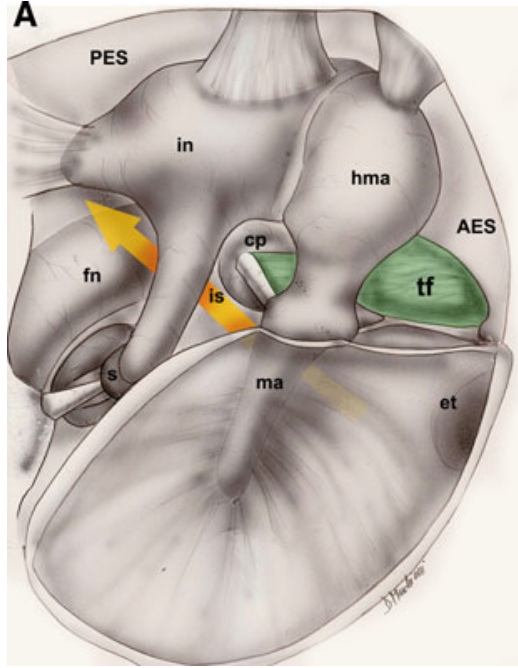

B

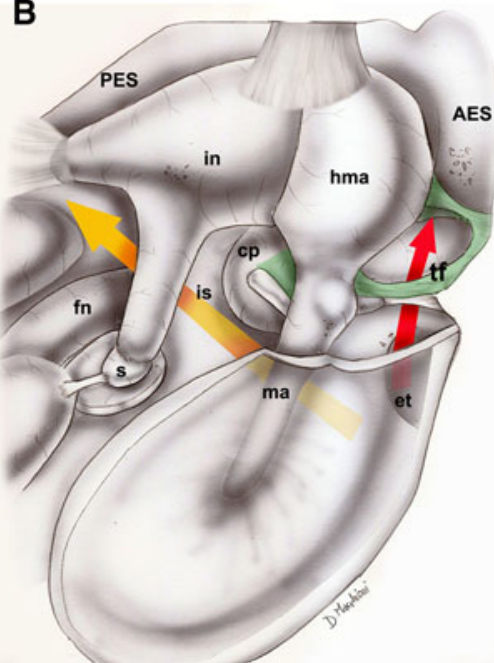

C

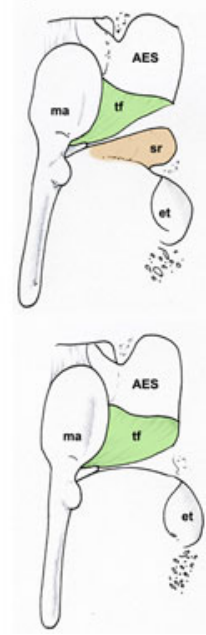

Fig. 12 Anatomy and variations of the tensor fold. a subject with a complete tensor fold separating AES from protympanum, the only aeration pathway to the epitympanum is through the isthmus (long arrow); b subject with an incomplete tensor fold, an additional ventilation route through the tensor fold is present in this case (short arrow); c1 Onal AES type I consisting of two cavities separated by the tensor tympani fold, the supratubal recess inferiorly and the

whereas the cavity inferior to it could be called the supratubal recess. AES type II (17\%) consists in one single cavity developing from the saccus anticus, in front of the cog, continuing with the eustachian tube anteriorly (Fig. 12c2). In subjects with the AES type I tensor fold is attached to a thick bony ridge anteriorly, which divides the AES two cavity (Fig. 12c1). The Authors also observed that for a good exposition of the AES using a microscope it was necessary to remove the incus and the head of the malleus with the cog. After these surgical maneuvers, during transmastoid approach for cholesteatoma surgery, is very important to remove also the tensor tympani fold and the supratubal ridge exposing the complete supratubal recess. In our experience, endoscopic approach permitted a direct exposition of the supratubal recess without drilling anterior malleal space superiorly. c2 Onal AES type II consisting of one single cavity anterior to the head of the malleus, in this case the supratubal recess is not present. ma Malleus, hma head of the malleus, in incus, $s$ stapes, $f n$ facial nerve, $P E S$ posterior epitympanic space, $A E S$ anterior epitympanic space, $t f$ tensor fold, et Eustachian tube, $c p$ cochlear process, is isthmus, $s r$ supratubal recess

any bone but just inserting a $45^{\circ}$ endoscope in the protympanic space (Fig. 13).

The supratubal recess is an independent area of variable size, which is related to inclination of the tensor fold. The more vertical is the tensor fold the wider is the supratubal recess. Endoscopic exploration of the AES (Onal's anterior mallear space) allows us to understand variations in size and shape of this anatomic region. Furthermore, AES can be composed by a single large air cell or by several small cells. In an unpublished study carried on in our department, we found that subjects affected by cholesteatoma limited to the attic show a reduced volume of bony boundaries of anterior epitympanum. If these findings will be confirmed, the little anterior epitympanic cavities might be a proof of a selective attic disventilation. 


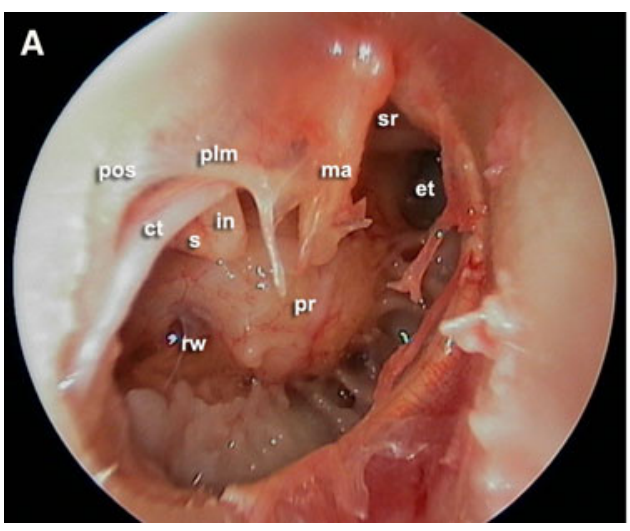

Fig. 13 Supratubal recess view with a $45^{\circ}$ endoscope in the protympanic space. a right ear with wide perforation of the drum; b magnification of the protympanum with a good view of the anatomic relationship between Eustachian tube and the supratubal

Tensor fold has a strategic position because it prevents communication between the sovratubaric recess, region belonging to the mesotympanum, and the overlying anterior epitympanum. When the tensor fold is complete (Fig. 12a) the only ventilation pathway to the AES is through the isthmus. According to Palva's studies the tensor fold is incomplete in only $25 \%$ of cases (Fig. 12b), allowing an alternative ventilation route from sovratubaric recess directly toward the attic (anterior epitympanum). The tensor fold has a very variable anatomy: in most subjects it shows a superior concavity, extending laterally from the semicanal of the tensor tympani muscle to the lateral aspect of protympanum, posteriorly adhering to the cochleariform process and to the tensor tympani tendon and extends anteriorly to the root of zygomatic bone becoming the epitympanic floor. When it inserts on the transverse crest its direction is almost vertical, while if it inserts on the tubaric tegmen its direction is rather horizontal.

Usually it has a curvature of $45^{\circ}$ and its most frequent insertion lies at the central portion of the anterior sovratubaric-epitympanic tegmen. According to our observations on patients affected by attic cholesteatomas, a complete tensor fold has been observed in almost all patients studied and the direction of the fold was in most cases horizontal. Moreover, we observed that in the peripheral portion the tensor fold presents a thicker component, whereas the central portion of the fold is usually more subtle and transparent and can be easily cut. Depending by its angle, the underlying sovratubaric recess is wider or smaller. The lateral part of the tensor fold keep close relationship with the most anterior portion of chorda tympani, which is parallel to the tensor tympani since it inserts anteriorly into the petrotympanic slot. The chorda tympani's fold inserts laterally onto the anterior malleolar ligament fold. Due to its anatomic characteristic the tensor

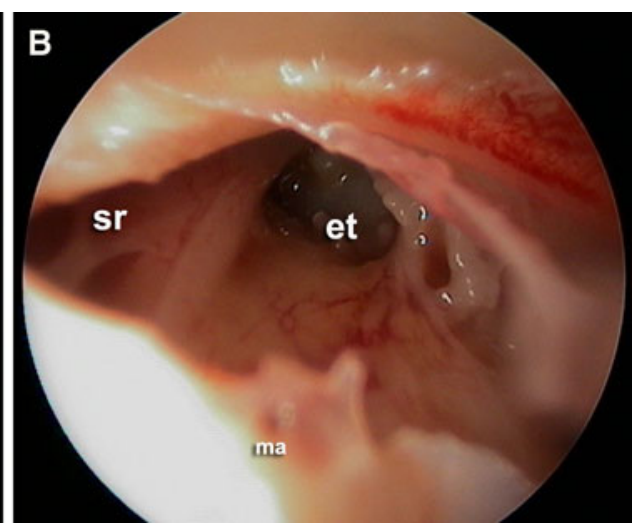

recess. ma Malleus, in incus, $s$ stapes, et Eustachian tube, sr supratubal recess, $c t$ corda tympani, pos posterior spine, $r w$ round window, $p r$ promontory, $p l m$ posterior malleal ligamental folds

fold region is extremely important in the middle-ear physiology, leading to a clear separation between anterior epitympanum and protympanum in terms of ventilation. When performing middle-ear surgery for a disventilation pathology with isthmus blockage, section of the central portion of the tensor fold is fundamental in order to create an alternative ventilation route between protympanum and AES.

Introduction of an endoscope during a transcanal approach allow the surgeon to have a direct vision of the anatomical structures presents in the AES. Using a $45^{\circ}$ optic surgeon has a wide view of the floor of the AES (cochleariform process and inferior edge of the tensor fold) without drilling any bone from the epitympanum and without removing the ossicular chain. If we remove the anterior portion of the scutum by drilling the bone under endoscopic control, we discover the incudo-malleolar joint and the AES. We used this approach to have a wider exposition of the superior edge of the tensor fold.

Endoscopic view allowed us to understand the complex anatomy of the epitympanic diaphragm and to describe the endoscopic anatomy of the tensor fold. In our experience the majority of subjects have a complete tensor fold separating the anterior epitympanum from the supratubal recess and protympanum space. The posterior-inferior border of this structure was attached to the tensor tympani tendon between the cochleariform process and the handle of the malleus. We found in these subjects a vertically orientation of the tensor fold. In few subjects we found a horizontal orientation of tensor fold with the anterior insertion over the eustachian tube. In these patients supratubal recess was not found.

Using a traditional microscopic surgery some authors $[28,29]$ have proposed different surgical approaches to visualize the tensor fold. Canal wall up techniques don't 
permit the visualization of the tensor fold, because the working angle was through the ear canal and the tensor fold remained hidden behind the neck of the malleus. For this reason, Morimitsu et al. [28] proposed an "anterior tympanotomy". During this approach the surgeon works parallel to the axis of the ear canal removing the bone from the lateral attic to the zygoma. This working axis allows him to drill in front of the malleus head removing the tensor fold.

Palva et al. strongly believes that the tensor fold removal during cholesteatoma surgery permit an additional aeration pathway to the attic, restoring epitympanic ventilation and preventing development of the retraction pocket and cholesteatoma recurrences in the postoperative time [25]. He suggested a microscopic endaural atticotomy extending to the supratubal recess and when ossicular chain is intact he cut the neck of the malleus to allow lateral lifting of the manubrium. This maneuver, exposing the tensor tendon, allowed a tensor fold removal.

We propose an endoscopic approach to the tensor fold. From our study transcanal endoscopic approach to the floor of the AES allowed a wider exposure of the anterior epitympanic spaces (supratubal recess and anterior mallear space) thus, permitting to highlight the tensor tympani fold. We suggested two kind of approach to remove this fold using an endoscope of $3 \mathrm{~mm}$ diameter $45^{\circ}$ as following [30]:

- Inferior approach. The endoscope is inserted in the protympanic region; we identify the Eustachian tube and supratubal recess. This position allows a good exposition of the inferior edge of the tensor fold.

- Superior approach. We perform an anterior atticotomy exposing the anterior epitympanic space. This procedure allows a good view on superior edge of tensor fold.

Especially the transcanal endoscopic approach permitted a good exposure of the tympanic isthmus using the $3 \mathrm{~mm} 0$ and $45^{\circ}$ endoscopes inserted into the tympanic cavity. With these optical instruments we are able to explore the large tympanic isthmus between the medial portion of the posterior incudal ligament posteriorly and the tensor tendon anteriorly. The $0^{\circ}$ endoscope allows us to magnify the space between the incudostapedial joint and cochleariform process with the tensor tendon (Proctor's anterior isthmus). After posterior atticotomy the $45^{\circ}$ endoscope permit us to magnify the space between the pyramidal process and the short process of the incus (Proctor's posterior isthmus).

\section{Protympanic Space}

The protympanic space is a pneumatic portion of the middle ear that lies anteriorly to the mesotympanum and inferiorly to the AES. The cochleariform process and the tensor fold with the tensor tympani canal represent the upper limit of protympanic space, while posteriorly its limit is generally considered the promontorium. Protympanic space is less important in middle ear surgery than other spaces, because chronic disease seldom involve this recess. Nonetheless some important structures are in there. Tympanic portion of Eustachian tube starts from the protympanum and is usually 11-12 mm in diameter. It can present different shapes: rectangular (35\%), triangular $(20 \%)$ of irregular shape $(45 \%)$ [31]. Above and medially to the Eustachian tube opening runs the internal carotid artery. Bone over this structure could be thick or pneumatized with some cells in there (protympanic cells). This variant is important because we can find a bulging of the carotid artery, which in some cases could be uncovered. Another important reason to explore this recess is that when we find protympanic cells in a patient with cholesteatoma disease involving the protympanic space, we have to pay more attention because these cells might hide the presence of cholesteatoma persistence.

\section{Conclusion}

Knowledge of middle ear anatomy is the basis for any otologist. Without a good knowledge of the anatomy is impossible to perform ear surgery and to understand ear physiology. Many dissection studies have been done in the past by many authors who described very well the bones structures in middle ear. Palva on the other hand mostly focused his attention to the tympanic folds and ventilation pathways. Based on our experience with endoscopic middle ear surgery we think that "in vivo" endoscopy of middle ear is the best way to explore the tympanic cavity and to understand the importance of ventilation routes, which we believe are the most important pathogenetic causes in chronic middle ear disease. Endoscopy allowed us to understand that ventilation in middle ear doesn't mean just Eustachian tube function, but also isthmus blockage, complete or incomplete tensor fold and mastoid pneumatization. If these theories will be confirmed in the future, endoscopic surgery will be a more physiologic and less invasive middle ear surgery. The goal of surgery in the chronic pathology of the middle ear should be restoration of normal ventilation of the attical-mastoid area. This is possible by removing the tensor fold and restoring the functionality of the isthmus.

These were the main reasons why we did a review of middle ear anatomy, especially focused on how it appears to the surgeon while performing an endoscopic procedure. 


\section{References}

1. Prevedello DM, Doglietto F, Jane JA Jr et al (2007) History of endoscopic skull base surgery: its evolution and current reality. J Neurosurg 107:206-213

2. Mouton WG, Bessell JR, Maddern GJ (1998) Looking back to the advent of modern endoscopy: 150th birthday of Maximilian Nitze. World J Surg 22:1256-1258

3. Reuter HJ (1988) Philipp Bozzini and endoscopy in the 19th Century. Max Nitze Museum, Stuttgart

4. Baki FA, El Dine MB, El Said L et al (2002) Sinus tympani endoscopic anatomy. Otolaryngol Head Neck Surg 127:158-162

5. Thomassin JM, Korchia D, Doris JM (1993) Endoscopic guided otosurgery in the prevention of residual cholesteatomas. Laryngoscope 103:939-943

6. Badr-El-Dine M (2002) Value of ear endoscopy in cholesteatoma surgery. Otol Neurotol 23:631-635

7. Tarabichi M (1997) Endoscopic management of acquired cholesteatoma. Am J Otol 18:544-549

8. Tarabichi M (2004) Endoscopic management of limited attic cholesteatoma. Laryngoscope 114:1157-1162

9. Bowdler DA, Walsh RM (1995) Comparison of the otoendoscopic and microscopic anatomy of the middle ear cleft in canal wall-up and canal wall-down temporal bone dissections. Clin Otolaryngol Allied Sci 20:418-422

10. Bottril ID, Poe DS (1995) Endoscope-assisted ear surgery. Am J Otol 16:158-163

11. Karhuketo TS, Puhakka HJ, Laippala PJ (1997) Endoscopy of the middle ear structures. Acta Otolaryngol Suppl 529:34-39

12. Ozturan O, Bauer CA, Miller CC et al (1996) Dimensions of the sinus tympani and its surgical access via a retrofacial approach. Ann Otol Rhinol Laryngol 105:776-783

13. Steinbrugge H (1889) On sinus tympani. Arch Otolaryngol 8: 53-57

14. Weiss MH, Parisier SC, Han JC et al (1992) Surgery for recurrent and residual cholesteatoma. Laryngoscope 102:145-151

15. Pulec JL (1996) Sinus tympani: retrofacial approach for the removal of cholesteatomas. Ear Nose Throat J 75:77-88

16. Jeng FC, Tsai MH, Brown CJ (2003) Relationship of preoperative findings and ossicular discontinuity in chronic otitis media. Otol Neurotol 24:29-32
17. Richards S, Kilby D (1971) Mastoidectomy using otoplastic flap. J Laryngol Otol 85:1007-1112

18. Pickett BP, Cail WS, Lambert PR (1995) Sinus tympani: anatomic considerations, computed tomography, and a discussion of the retrofacial approach for removal of disease. Am J Otol 16: $541-550$

19. El-Meselaty K, Badr-El-Dine M, Mandour M et al (2003) Endoscope affects decision making in cholesteatoma surgery. Otolaryngol Head Neck Surg 129:490-496

20. Holt JJ (2005) The ponticulus: an anatomic study. Otol Neurotol 26:1122-1124

21. Presutti L, Marchioni D, Mattioli F, Villari D, Alicandri Ciufelli M (2008) Endoscopic management of acquired cholesteatoma: our experience. J Otolaryngol 4:1-7

22. Pratt LL (1984) Complications associated with the surgical treatment of cholesteatoma. Laryngoscope 93:289-291

23. Marchioni D, Mattioli F, Alicandri-Ciufelli M et al (2009) Transcanal endoscopic approach to the sinus tympani: a clinical report. Otol Neurotol 30(6):758-765

24. Marchioni D, Alicandri-Ciufelli M, Grammatica A et al (2010) Pyramidal eminence and subpyramidal space: an endoscopic anatomical study. Laryngoscope 120(3):557-564

25. Palva T, Johnsson L (1995) Epitympanic compartment surgical considerations: reevaluation. Am J Otol 16(4):505-513

26. Palva T, Ramsay H (1996) Incudal folds and epitympanic aeration. Am J Otol 17:700-708

27. Onal K, Van Haastert RM, Grote JJ (1997) Structural variations of supratubal recess: the anterior epitympanic space. Am J Otol $18: 317-321$

28. Farrior JB (1968) Tympanoplasty: the anterior attico-tympanotomy. Surgery of the posterior tympanic recess. Laryngoscope 78:768-779

29. Donaldson JA, Anson BJ, Warphea RL et al (1970) The surgical anatomy of the sinus tympani. Arch Otolaryngol 91:219-227

30. Marchioni D, Mattioli F, Alicandri-Ciufelli M et al (2008) Endoscopic approach to tensor fold in patients with attic cholesteatoma. Acta Otolaryngol 25:1-9

31. Savic D, Djeric D (1985) Anatomical variations and relations in the medial wall of the bony portion of the eustachian tube. Acta Otolaryngol 99(5-6):551-556 